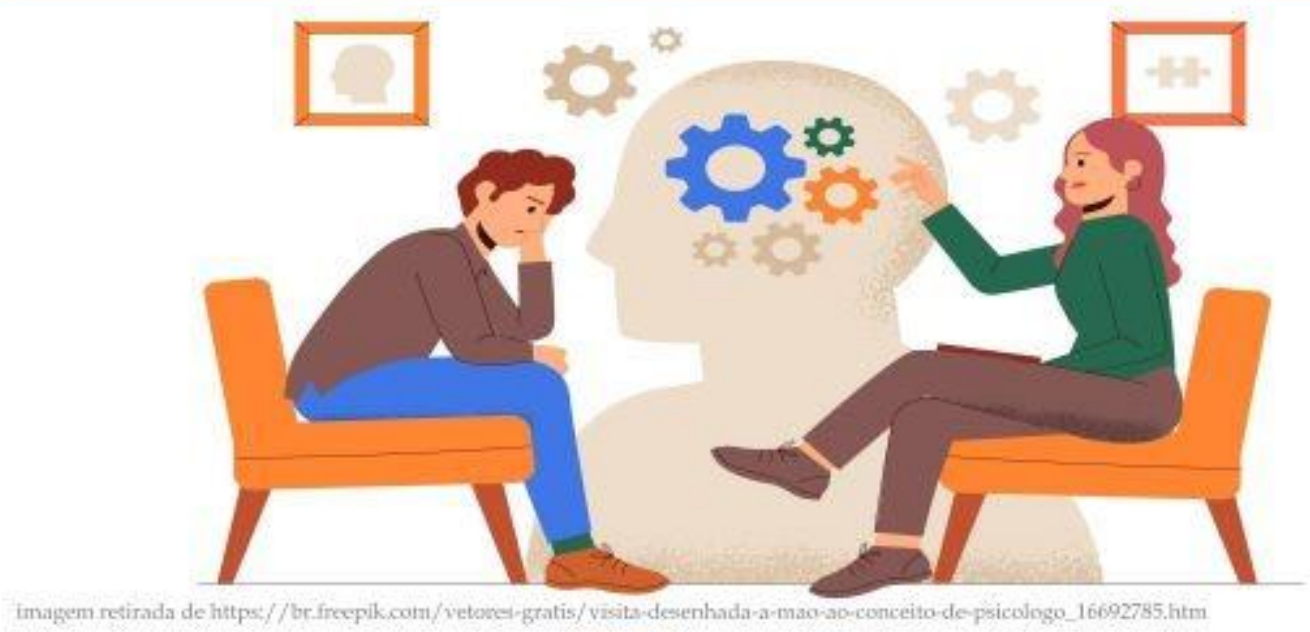

\title{
CONTRIBUIÇÕES DA TERAPIA COGNITIVO-COMPORTAMENTAL NO TRATAMENTO DA DISFUNÇÃO TEMPOROMANDIBULAR (DTM)
}

\author{
Hellorainy Rangel Rodrigues de Souza \\ ORCID: https://orcid.org/0000-0002-0620-1220. \\ E-mail: hellorainysouza@id.uff.br. \\ Ana Lúcia Novais Carvalho \\ ORCID: https://orcid.org/0000-0002-4808-8449. \\ E-mail: analucianovaiscarvalho@id.uff.br.
}

Resumo: A Disfunção Temporomandibular (DTM) é um distúrbio que afeta as articulações temporomandibulares e os músculos mastigatórios, entre outras estruturas da face. Os sintomas podem gerar dores nessas articulações e músculos, bem como dores na cabeça, orelha, ruídos nas articulações, zumbidos, dores no ouvido, limitação na abertura da boca, entre outros. Sua etiologia é multifatorial. Entre os fatores que podem influenciar a DTM encontram-se os psicológicos. Assim, este estudo teve como objetivo identificar as principais estratégias da terapia cognitivo-comportamental (TCC) utilizadas para o tratamento de indivíduos diagnosticados com DTM. Por método, adotou-se a revisão da literatura. Os bancos de dados utilizados foram PePSIC, SCIELO, PubMed e BVS, em inglês e português. Os descritores utilizados em todos os bancos foram: DTM e terapia cognitivacomportamental; Disfunção temporomandibular e terapia-cognitiva comportamental, Disfunção temporomandibular e terapia-cognitiva, TMD and cognitive behavioral therapy; Temporomandibular disorder and cognitive behavioral therapy, Temporomandibular disorder and cognitive therapy. Os filtros utilizados para inclusão dos estudos foram artigos sobre intervenções em TCC, em português e/ou inglês e com datas de publicação dos últimos três anos. Seis artigos foram analisados, após a passagem dos filtros. Os resultados apontam que as principais estratégias de TCC utilizadas no tratamento da DTM são a restruturação cognitiva, a autogestão, a psicoeducação e o relaxamento. Sugerem, também, que a TCC pode trazer contribuições positivas para os casos de DTM, porém a amostra deste estudo foi pequena. Os artigos analisados apontam que o tratamento multidisciplinar deve incluir intervenções cognitivas e comportamentais. Sendo assim, fica evidenciada a necessidade de realização de mais pesquisas com o objetivo de verificar os efeitos da TCC em pacientes com DTM, principalmente, estudos com ensaios clínicos.

Palavras-chave: Terapia cognitiva-comportamental. Disfunção temporomandibular. DTM. Estresse. Ansiedade.

\section{POLÊM!CA | LABORE (3)}

Polêmica - Revista Eletrônica da Uerj - Rua São Francisco Xavier, 524, $1^{\circ}$ andar bloco D, sl.1001 • Tels.: +55 21 2334-4088 / 4087 • http://www.e-publicacoes.uerj.br/index.php/polemica/index http://www.labore.uerj.br • laboreuerj@yahoo.com.br 


\title{
CONTRIBUTIONS OF COGNITIVE BEHAVIORAL THERAPY IN THE TREATMENT OF TEMPOROMANDIBULAR DISORDERS (TMD)
}

\begin{abstract}
Temporomandibular Disorder (TMD) is a disorder that affects the temporomandibular joints and masticatory muscles, among other facial structures. Symptoms can generate pain in these joints and muscles, as well as pain in the head, ear, noise in the joints, tinnitus, ear pain, limitation in opening the mouth, among others. Its etiology has multifactorial causes. Among the factors that can influence TMD are psychological. Objective: This study aimed to identify cognitive behavioral therapy (CBT) strategies used to treat individuals diagnosed with TMD. Method: Literature review. The databases used were PePSIC, SCIELO, PubMed and BVS, in English and Portuguese. The descriptors used in databases were: TMD and cognitive-behavioral therapy; Temporomandibular disorders and cognitive behavioral therapy, Temporomandibular disorders and cognitive therapy, TMD and cognitive behavioral therapy; Temporomandibular disorder and cognitive behavioral therapy, Temporomandibular disorder and cognitive therapy. The filters used to include the studies were: articles on CBT interventions, articles in Portuguese and/or English and with publication dates in the last three years. Results: Six articles were analyzed, after passing the filters. Discussion: The results show that the main CBT guidelines used in the treatment of TMD are: cognitive restructuring, self-management, psychoeducation and relaxation training. They also suggest that a CBT can bring positive contributions to TMD cases, but the sample in this study was small. The articles point out that multidisciplinary treatment must include cognitive and behavioral interventions. Thus, the need for further research to verify the effects of CBT in patients with TMD is evidenced, especially, studies with clinical trials.

Keywords: Cognitive behavioral therapy. Temporomandibular disorder. TMD. Stress. Anxiety.
\end{abstract}

\section{Introdução}

A disfunção temporomandibular (DTM) é descrita pela Academia Americana de Dor Orofacial como sendo um distúrbio que afeta as articulações temporomandibulares e os músculos mastigatórios, entre outras estruturas da face, fazendo com que o sujeito sinta dores nessas regiões dos músculos e articulações (LEEUW, 2010 apud CARRARA et al., 2010, p. 115). Portero et al. (2009) salientam que as disfunções podem ser classificadas entre desordens da articulação temporomandibular (ATM), desordens dos músculos mastigadores, doenças congênitas e do desenvolvimento.

Dores de ouvido, dores de cabeça, enxaqueca e presença de ruídos nas articulações são sintomas e sinais de DTM, segundo Portero et al. (2009). Rota (2019) aponta, ainda, a presença de sintomas como zumbidos, sensação de plenitude auricular, vertigens, dificuldade nos movimentos para abrir e fechar a boca e há a possibilidade de ocorrer sensibilidade da musculatura e da ATM. Okeson (2013) enfatiza que pode ocorrer, também, o travamento da mandíbula em determinadas situações.

A prevalência de sintomas de DTM é maior em indivíduos na faixa etária entre 20 e 45 anos de idade. Nos idosos a causa mais relacionada a essa disfunção é o desgaste da articulação (LIU; STEINKELER, 2013 apud STÜERMER, 2020, p. 13-14). Em adolescentes a presença de sintomas de ansiedade é um importante fator de risco para o desenvolvimento desta patologia (MOTTA et al., 2015). Rosal (2019) salienta que também é possível da DTM ocorrer em

\section{POLÊM!CA $\mid$ LABORE}

Polêmica - Revista Eletrônica da Uerj - Rua São Francisco Xavier, 524, $1^{\circ}$ andar bloco D, sl.1001 • Tels.: +55 21 2334-4088 / 4087 • http://www.e-publicacoes.uerj.br/index.php/polemica/index http://www.labore.uerj.br • laboreuerj@yahoo.com.br 
crianças, todavia sua etiologia estaria mais relacionada a fatores emocionais e ao mau encaixe dos dentes. Esses autores ainda mencionam a importância do diagnóstico ser precoce, pois os sintomas tendem a se acentuar com o aumento da idade.

Em relação ao sexo, a incidência de DTM é maior em indivíduos do sexo feminino (MARTINS, 2006).

Sobre a etiologia da DTM, Okeson (2013) e Biasotto-Gonzalez (2005) esclarecem que é difícil apontar uma causa exata para o surgimento desta patologia. Luna et al. (2015) destacam que a etiologia se refere a três fatores principais, sendo eles: neuromusculares, psíquicos e anatomo-oclusais. Sendo assim, a DTM pode ocorrer e ser mantida por diversos fatores como trauma, hábitos parafuncionais, desalinhamento dentário, abertura bucal em excesso, doenças sistêmicas, posturas inadequadas e fatores emocionais (PORTERO et al., 2009).

Como destacado por Ferreira et al. (2009), corroborando com Massena e Frassetto (2015), os aspectos psicológicos nas disfunções têm sido apontados como condições importantes na etiologia. Nesse sentido, a ansiedade, o estresse, as características da personalidade e a depressão são os fatores psicológicos mais associados à DTM.

Considerando o papel de fatores psicológicos na etiologia da DTM, o presente estudo tem como objetivo identificar as principais estratégias da terapia cognitivo-comportamental (TCC) utilizadas para o tratamento de indivíduos diagnosticados com DTM.

\section{Metodologia}

O presente trabalho trata de um estudo de revisão da literatura.

A amostra foi constituída por artigos que apresentavam intervenções da TCC realizadas em pacientes com DTM. A busca dos artigos ocorreu por meio de consultas nas bases de dados da PePSIC, SCIELO, PubMed e BVS no período do dia 15 a 20 de julho do ano de 2021.

Os descritores utilizados em todas as bases de dados foram, em português: DTM AND terapia cognitiva-comportamental; Disfunção temporomandibular AND terapia cognitivacomportamental; Disfunção temporomandibular AND terapia-cognitiva; e em inglês: TMD AND cognitive behavioral therapy; Temporomandibular disorder AND cognitive behavioral therapy; Temporomandibular disorder and cognitive therapy.

Foram incluídos os artigos que obedeciam aos seguintes critérios: a) ser um artigo que abordasse sobre a intervenção da TCC em pacientes com DTM; b) ter sido publicado nos

\section{POLÊM!CA | LABORE}

Polêmica - Revista Eletrônica da Uerj - Rua São Francisco Xavier, 524, $1^{\circ}$ andar

bloco D, sl.1001 • Tels.: +55 21 2334-4088 / 4087 • http://www.e-publicacoes.uerj.br/index.php/polemica/index http://www.labore.uerj.br • laboreuerj@yahoo.com.br 
idiomas português/inglês; c) ter sido realizado entre os anos de 2018 e 2020. Depois da aplicação dos filtros nos artigos, passou-se para a etapa da análise qualitativa dos trabalhos.

\section{Resultados}

A partir do refinamento das buscas, com base no critério de inclusão e exclusão, restaram 6 artigos, sendo descritas abaixo as características dos estudos (Tabela 1) e as intervenções aplicadas (Tabela 2).

Tabela - 1: Informações gerais

\begin{tabular}{|c|c|c|c|c|}
\hline Autor e ano & Objetivo & Metodologia & Participantes & Resultados/Conclusões \\
\hline $\begin{array}{l}\text { Zhang et al. } \\
(2020)\end{array}$ & $\begin{array}{l}\text { Aplicar } \\
\text { intervenções da } \\
\text { terapia cognitiva- } \\
\text { comportamental } \\
\text { em pacientes com } \\
\text { DTM. }\end{array}$ & $\begin{array}{l}\text { Ensaio clínico } \\
\text { randomizado }\end{array}$ & $\begin{array}{l}108 \\
\text { participantes }\end{array}$ & $\begin{array}{l}\text { Os resultados ainda não } \\
\text { foram divulgados. }\end{array}$ \\
\hline $\begin{array}{l}\text { Takeuchi- } \\
\text { Sato et al. } \\
\text { (2020) }\end{array}$ & $\begin{array}{l}\text { Avaliar o sistema } \\
\text { de registro e } \\
\text { lembrete por e-mail } \\
\text { para contato } \\
\text { dentário não } \\
\text { funcional durante o } \\
\text { dia (NFTC*) em } \\
\text { pacientes com } \\
\text { DTM. }\end{array}$ & Estudo de caso & 30 participantes & $\begin{array}{l}\text { O sistema de lembrete e } \\
\text { registro de NFTC pode } \\
\text { ser considerado como } \\
\text { uma ferramenta de } \\
\text { autogerenciamento } \\
\text { eficaz para corrigir e } \\
\text { controlar o NFTC } \\
\text { diurno, bem como } \\
\text { auxiliar no tratamento. } \\
\text { Além disso, foi possível } \\
\text { perceber que o sistema } \\
\text { de gravação foi mais } \\
\text { eficaz do que a TCC } \\
\text { convencional, no que se } \\
\text { refere à diminuição do } \\
\text { NFTC diurno. }\end{array}$ \\
\hline $\begin{array}{l}\text { Lan et al. } \\
(2020)\end{array}$ & $\begin{array}{l}\text { Avaliar a eficácia } \\
\text { do programa } \\
\text { guiado de dor } \\
\text { multimodal } \\
\text { baseado na internet } \\
\text { para adultos com } \\
\text { dor crônica devido } \\
\text { à DTM em } \\
\text { comparação com a } \\
\text { terapia de placa } \\
\text { oclusal } \\
\text { convencional. }\end{array}$ & $\begin{array}{l}\text { Ensaio clínico } \\
\text { randomizado }\end{array}$ & 43 participantes & $\begin{array}{l}\text { Não houve diferença de } \\
\text { resultado entre o } \\
\text { programa de dor } \\
\text { multimodal baseado na } \\
\text { internet (que incluía a } \\
\text { terapia cognitivo- } \\
\text { comportamental e } \\
\text { princípio de autogestão) } \\
\text { e a terapia da placa } \\
\text { oclusal. }\end{array}$ \\
\hline $\begin{array}{l}\text { Nagata et al. } \\
\text { (2019) }\end{array}$ & $\begin{array}{l}\text { Avaliar a eficácia } \\
\text { do uso da } \\
\text { manipulação, }\end{array}$ & $\begin{array}{l}\text { Ensaio clínico } \\
\text { randomizado }\end{array}$ & 61 participantes & $\begin{array}{l}\text { Não houve diferença } \\
\text { estatisticamente } \\
\text { significativa entre os }\end{array}$ \\
\hline
\end{tabular}

\section{POLÊM!CA $\mid$ LABORE}

Polêmica - Revista Eletrônica da Uerj - Rua São Francisco Xavier, 524, $1^{\circ}$ andar bloco D, sl.1001 • Tels.: +55 21 2334-4088 / 4087 • http://www.e-publicacoes.uerj.br/index.php/polemica/index http://www.labore.uerj.br • laboreuerj@yahoo.com.br 


\begin{tabular}{|c|c|c|c|c|}
\hline & $\begin{array}{l}\text { associada ao } \\
\text { tratamento } \\
\text { tradicional, em } \\
\text { pacientes com } \\
\text { DTM. }\end{array}$ & & & $\begin{array}{l}\text { grupos: tratamento } \\
\text { convencional e } \\
\text { tratamento convencional } \\
\text { mais manipulação. } \\
\text { Tratamento } \\
\text { convencional inclui TCC } \\
\text { para bruxismo e } \\
\text { psicoeducação. }\end{array}$ \\
\hline $\begin{array}{l}\text { Cole \& } \\
\text { Carlson } \\
(2018)\end{array}$ & $\begin{array}{l}\text { Descrever os } \\
\text { fatores cognitivos, } \\
\text { comportamentais e } \\
\text { emocionais que } \\
\text { contribuem para o } \\
\text { início e } \\
\text { manutenção das } \\
\text { DTMs crônicas. } \\
\text { Discutir o } \\
\text { tratamento } \\
\text { cognitivo e } \\
\text { comportamental } \\
\text { das DTMs. }\end{array}$ & $\begin{array}{l}\text { Revisão de } \\
\text { literatura }\end{array}$ & Não se aplica & $\begin{array}{l}\text { O aumento da } \\
\text { consciência somática, as } \\
\text { emoções negativas e o } \\
\text { estresse podem ser } \\
\text { fatores de risco para o } \\
\text { desenvolvimento de } \\
\text { DTM. Além disso, } \\
\text { fatores comportamentais } \\
\text { como sono insatisfatório, } \\
\text { comportamentos } \\
\text { parafuncionais } \\
\text { frequentes e história de } \\
\text { tabagismo podem } \\
\text { também influenciar nas } \\
\text { DTMs. Diante disso, o } \\
\text { tratamento precisa } \\
\text { abordar as distorções } \\
\text { cognitivas, } \\
\text { autorregulação e } \\
\text { gerenciamento } \\
\text { comportamental. }\end{array}$ \\
\hline $\begin{array}{l}\text { Noma et al. } \\
(2020)\end{array}$ & $\begin{array}{l}\text { Estudar o uso da } \\
\text { TCC para dor } \\
\text { orofacial. }\end{array}$ & $\begin{array}{l}\text { Revisão } \\
\text { narrativa da } \\
\text { literatura }\end{array}$ & Não se aplica & $\begin{array}{l}\text { A TCC traz resultados } \\
\text { positivos para a dor } \\
\text { orofacial. Quando } \\
\text { combinada com o uso de } \\
\text { aparelhos intraorais, } \\
\text { gerenciamento do } \\
\text { estresse e biofeedback, a } \\
\text { TCC pode ser útil para o } \\
\text { tratamento da disfunção } \\
\text { temporomandibular. }\end{array}$ \\
\hline
\end{tabular}

*NFTC - contato dentário não funcional durante o dia.

Fonte: As autoras (2021).

A partir da análise dos artigos foi possível identificar os principais procedimentos técnicos utilizados nos estudos.

\section{POLÊM!CA $\mid$ LABORE}

Polêmica - Revista Eletrônica da Uerj - Rua São Francisco Xavier, 524, $1^{\circ}$ andar bloco D, sl.1001 • Tels.: +55 21 2334-4088 / 4087 • http://www.e-publicacoes.uerj.br/index.php/polemica/index http://www.labore.uerj.br • laboreuerj@yahoo.com.br 
Tabela - 2: Intervenções/Técnicas utilizadas

\begin{tabular}{ll}
\hline Autor e ano & Intervenção \\
\hline Zhang et al. (2020) & Grupo controle: Intervenção convencional com uso de talas \\
& oclusais, medicamentos anti-inflamatórios não esteroides, \\
& educação do paciente, exercícios de movimento mandibular, \\
& modificação de hábitos parafuncionais e/ou dietéticos e aplicação \\
& regular de frio e calor. \\
& Grupo experimental: Intervenção com procedimentos da TCC. \\
& Três estágios de intervenções, com duração de 30 minutos. \\
& Nessas intervenções foi usado o estabelecimento de um bom \\
& relacionamento com o paciente, estabelecimento de metas, \\
& avaliação cognitiva, identificação do conteúdo cognitivo e \\
& reestruturação cognitiva. \\
& Grupo-e: Os pacientes foram lembrados de evitar o NFTC pelo \\
& sistema de gravação e por lembrete por meio do e-mail. \\
& Grupo-s: Os pacientes receberam a autogestão da TCC e \\
& instruções em lembretes de nota adesiva por 20 dias, para seus \\
Takeuchi-Sato et al. (2020) & computadores, televisores e estantes de livros. \\
& Grupo controle: Os participantes receberam instruções verbais \\
& simples para manterem os dentes separados sempre que \\
percebessem o contato não funcional. \\
As instruções foram fornecidas antes do período experimental e \\
nenhuma instrução foi repetida depois. \\
Não se aplica.
\end{tabular}

Cole \& Carlson (2018)

Lan et al. (2020)
Não se aplica.

Estudo randomizado: Programa com 7 módulos de tratamento de dor multimodal baseado na internet, com princípios da terapia cognitiva-comportamental e autogerenciamento.

Módulo 1: Informações gerais. Reflexão sobre estratégias anteriores de tratamento para dor na DTM. Intervenções na TCC: Fornecimento de valores e objetivos.

Módulo 2: Informações sobre a etiologia e epidemiologia da dor na DTM. Intervenções na TCC: psicoeducação e avaliação. Módulo 3: informações sobre os fatores que mantêm a dor na DTM, a fisiologia da dor, dor aguda e crônica e a relação entre qualidade de vida e DTM. Intervenções na TCC: psicoeducação e avaliação.

Módulo 4: Informações sobre alternativas de tratamento. Intervenção na TCC: técnica de relaxamento aplicado e treinamento de habilidades.

Módulo 5: Informações a respeito do estresse e ligação dele com a dor. Intervenções na TCC: análise funcional, resolução de problemas, relaxamento, treinamento de habilidades.

Módulo 6: Informações sobre o gerenciamento do estresse, dor, exercício respiratório, dieta, sono, treinamento, gerenciamento do tempo, estabelecimento de limites e aceitação, relaxamento contínuo e exercícios mandibulares. Intervenção na TCC: ação comprometida, relaxamento e treinamento de habilidades.

\section{POLÊM!CA $\mid$ LABORE}

Polêmica - Revista Eletrônica da Uerj - Rua São Francisco Xavier, 524, $1^{\circ}$ andar bloco D, sl.1001 • Tels.: +55 21 2334-4088 / 4087 • http://www.e-publicacoes.uerj.br/index.php/polemica/index http://www.labore.uerj.br • laboreuerj@yahoo.com.br 
Módulo 7: Informações sobre o retrocesso, planejamento de manutenção, plano de manutenção e resumo do programa. Intervenções na TCC: resumo e plano de manutenção. Grupo controle: Tratamento convencional, com a utilização de tala de estabilização rígida do tipo Michigan colocada na parte superior da mandíbula.

Nagata et al. (2019) Grupo tratamento convencional.

Grupo tratamento convencional mais manipulação.

O tratamento convencional inclui TCC para bruxismo e psicoeducação.

Como o objetivo do estudo não foi avaliar o papel da TCC, ambos os grupos passaram por este tipo de intervenção.

Fonte: As autoras (2021).

\section{Discussão}

Os procedimentos técnicos da psicoterapia cognitivo-comportamental mais utilizados nos artigos pesquisados foram: psicoeducação, autogestão, relaxamento e reestruturação cognitiva. No estudo de Lan et al. (2020) alguns outros procedimentos foram identificados, como o uso da análise funcional, o treinamento de habilidades e a resolução de problemas.

Os resultados do estudo de Zhang et al. (2020) ainda não foram publicados, sendo possível apenas identificar as estratégias de TCC propostas: o estabelecimento de um bom relacionamento com o paciente, estabelecimento de metas, avaliação cognitiva, identificação do conteúdo cognitivo e reestruturação cognitiva.

No estudo de Lan et al (2020), o objetivo era comparar uma intervenção baseada em um programa para manejo da dor pela internet, que utilizava estratégias da TCC, com o tratamento convencional com placas oclusais. Os resultados não demonstraram diferenças significativas. Entretanto, no grupo do programa multimodal houve uma melhora na função da mandíbula. Os autores sugerem a realização de mais estudos.

No estudo de Takeuchi-Sato et al. (2020), embora tenha citado a utilização da TCC no tratamento do grupo experimental, as intervenções não foram bem esclarecidas. A única informação contida foi sobre a autogestão baseada na TCC, que seria benéfica para a DTM originada a partir das atividades parafuncionais diurnas. Os resultados sugeriram que o sistema de registro por e-mail foi mais eficaz que a TCC. O estudo apontou como limitação a não classificação da dor da DTM e a gravação ter ocorrido fora de ambientes laboratoriais.

O estudo de Nagata et al. (2019) não teve como objetivo avaliar a eficácia da TCC, desta forma, as técnicas desta terapia foram utilizadas nos dois grupos do estudo. Não houve diferença

\section{POLÊM!CA $\mid$ LABORE}

Polêmica - Revista Eletrônica da Uerj - Rua São Francisco Xavier, 524, $1^{\circ}$ andar bloco D, sl.1001 • Tels.: +55 21 2334-4088 / 4087 • http://www.e-publicacoes.uerj.br/index.php/polemica/index http://www.labore.uerj.br • laboreuerj@yahoo.com.br 
estatisticamente significativa entre os grupos: tratamento convencional e tratamento convencional mais manipulação. Tratamento convencional inclui TCC para bruxismo e psicoeducação.

Lan et al. (2020) e Nagata et al. (2019) incluíram como intervenção a psicoeducação. Esses autores apontaram, principalmente, para a necessidade de orientar os pacientes em relação ao estresse e à dor. Noma et al. (2020) também destacaram a importância do gerenciamento do estresse. O estresse é um fator que contribui para os sintomas da DTM (OKESON, 2013). Desta forma, estes autores, ao destacarem a importância de trabalhá-lo durante o tratamento, corroboraram com o que foi evidenciado na literatura pelos autores Graciola e Silveira (2013) Okeson (2013), Tosato (2011) e Urbani et al. (2019), sobre a influência do estresse na DTM.

Lan et al. (2020) também apontaram para intervenções contendo o autogerenciamento dos pacientes. Isso corrobora com Takeuchi-Sato et al. (2020), que também descreveram como intervenção a autogestão.

Os demais artigos foram estudos de revisão da literatura (NOMA et al., 2020; COLE; CARLSON, 2018). Os estudos abordaram intervenções na TCC para pacientes com DTM ou dor orofacial oriunda da DTM.

Okeson (2013) e Biasotto-Gonzalez (2005) destacaram que a DTM tem causas multifatoriais. Corroborando com essa perspectiva, Cole e Carlson (2018) salientaram, em sua revisão de literatura, que a DTM é complexa e a dor traz danos no nível biopsicossocial. Por isso, ficou evidenciado neste estudo que a intervenção precisa ser multiprofissional.

Noma et al. (2020), Zhang et al. (2020), Lan et al. (2020) e Cole e Carlson (2018) ressaltaram o uso de intervenção com a técnica de relaxamento. Também destacaram a importância de se usar como técnica a reestruturação cognitiva. Uma justificativa para o uso dela é que quando o paciente faz interpretações sobre a dor como sendo perigosa ou debilitante, há a tendência de causar mais sofrimentos psicológicos (NOMA et al., 2020).

Outra questão apontada por Noma et al. (2020) é a importância de que os dentistas considerem a relevância da inclusão da TCC no manejo da DTM. Isso corrobora com Zavanelli et al. (2017), que enfatizam sobre o benefício da TCC, que auxilia em uma melhor qualidade de vida para pacientes com DTM, e também com Braga e Souza (2016), que destacam sobre a importância dos dentistas não usarem só o tratamento convencional para a disfunção, mas incluírem as intervenções que abarquem outros fatores que a influenciam.

\section{POLÊM!CA | LABORE}




\section{Considerações finais}

Como foi possível observar durante a realização deste trabalho, a etiologia da DTM é considerada complexa; logo, não há como apontar uma causa específica. São diversos os fatores que podem contribuir para o seu surgimento e manutenção. Por isso, as intervenções precisam abarcar as questões biopsicossociais. Dentre os aspectos que influenciam a DTM, destacam-se os fatores psicológicos, sendo a ansiedade e o estresse como um dos principais que atuam na manutenção da disfunção.

Uma das abordagens da psicologia que vem contribuindo para o manejo da DTM é a terapia cognitiva-comportamental. Os resultados demonstraram que a TCC pode ser benéfica. Em geral, os artigos analisados apontaram a importância de técnicas, como a reestruturação cognitiva, a autogestão, a psicoeducação e o relaxamento. Assim sendo, são necessárias mais pesquisas que abordem o uso da TCC em pacientes com DTM, principalmente, ensaios clínicos.

\section{Referências}

BIASOTTO-GONZALEZ, D. A. Abordagem interdisciplinar das disfunções temporomandibulares. São Paulo: Editora Manole, 2005.

BRAGA, A. C.; SOUZA, F. D. Transtornos psicológicos associados à disfunção temporomandibula. Psicologia e Saúde em debate, v. 2, n. 1, p. 100-120, 2016. Disponível em: http://psicodebate.dpgpsifpm.com.br/index.php/periodico/article/view/31. Acesso em: 5 nov. 2021.

CARRARA, S. V.; CONTI, P. C. R.; BARBOSA, J. S. Termo do $1^{\circ}$ consenso em disfunção temporomandibular e dor orofacial. Dental Press Journal of Orthodontics, v. 15, n. 3, p. 114-120, 2010. DOI: https://doi.org/10.1590/S2176-94512010000300014.

COLE, H. A.; CARLSON, C. R. Mind-Body Considerations in Orofacial Pain. Dental clinics of North America, v. 62, n. 4, p. 683-694, out. 2018. Disponível em: https://www.sciencedirect.com/science/article/pii/S001185321830051X. Acesso em: 5 nov. 2021.

FERREIRA, K. D. M.; GUIMARÃES, J. P.; BATISTA, C. H. T.; JÚNIOR, A. M. L. F.; FERREIRA, L. A. Fatores psicológicos relacionados à sintomatologia crônica das desordens temporomandibulares - revisão de literatura. Revista da Faculdade de Odontologia - UPF, v. 14, n. 3, ago. 2010. Disponível em: http://seer.upf.br/index.php/rfo/article/view/796. Acesso em: 5 nov. 2021.

GRACIOLA, J.; SILVEIRA, A. M. Avaliação da influência do estresse na prevalência de disfunções temporomandibulares em militares estaduais do Rio Grande do Sul. Journal of Oral Investigations, v. 2, n. 1, p. 32-37, 2013. DOI: http://dx.doi.org/10.18256/2238-510X/j.oralinvestigations.v2n1p32-37.

LAN, J.; SVENSSON, P.; ALSTERGREN, P. Internet-Based Multimodal Pain Program with telephone support for adults with Chronic Temporomandibular Disorder Pain: randomized controlled pilot trial. Journal of Medical Internet Research, v. 22, n. 10, out. 2020. Disponível em: https://pubmed.ncbi.nlm.nih.gov/33048053/. Acesso em: 5 nov. 2021.

LUNA, I. M.; BARBOSA, M. A.; BITU, V. D. C. N. A ansiedade como fator etiológico das Disfunções Temporomandibulares. Revista Interfaces: Saúde, Humanas e Tecnologia, v. 3, n. 8, 2015. Disponível em:

\section{POLÊM!CA $\mid$ LABORE}

Polêmica - Revista Eletrônica da Uerj - Rua São Francisco Xavier, 524, $1^{\circ}$ andar bloco D, sl.1001 • Tels.: +55 21 2334-4088 / 4087 • http://www.e-publicacoes.uerj.br/index.php/polemica/index http://www.labore.uerj.br • laboreuerj@yahoo.com.br 
http://interfaces.leaosampaio.edu.br/index.php/revista-interfaces/article/view/489. Acesso em: 5 nov. 2021.

MARTINS, R. J. Disfunção temporomandibular: relação com a classe sócio-econômica, qualidade do sono e estresse. 2006. 130 f. Tese (Doutorado em Odontologia) - Universidade Estadual Paulista, Faculdade de Odontologia de Araçatuba, Araçatuba, 2006. Disponível em: http://hdl.handle.net/11449/104211. Acesso em: 5 nov. 2021.

MASSENA, P.; FRASSETTO, S. S. Aspectos psicológicos associados à disfunção temporomandibular: uma revisão sistemática da literatura. Aletheia, Canoas, n. 47-48, p. 169-182, dez. 2015. Disponível em: http://pepsic.bvsalud.org/scielo.php?script=sci_arttext\&pid=S1413-03942015000200014\&lng=pt\&nrm=iso. Acesso em: 5 nov. 2021.

MOTTA, L. J.; BUSSADORI, S. K.; GODOY, C. L. H. D.; BIAZOTTO-GONZALEZ, D. A.; MARTINS, M. D.; SILVA, R. S. Disfunção temporomandibular segundo o nível de ansiedade em adolescentes. Psicologia: Teoria e Pesquisa, v. 31, n. 3, p. 389-395, jul./set. 2015. DOI: https://doi.org/10.1590/010237722015031899389395.

NAGATA, K.; HORI, S.; MIZUHASHI, R.; YOKOE, T.; ATSUMI, Y.; NAGAI, W.; GOTO, M. Efficacy of mandibular manipulation technique for temporomandibular disorders patients with mouth opening limitation: a randomized controlled trial for comparison with improved multimodal therapy. Journal of Prosthodontic Research, v. 63, n. 2, p. 202-209, abr. 2019. DOI: https://doi.org/10.1016/j.jpor.2018.11.010.

NOMA, N.; WATANABE, Y.; SHIMADA, A.; USUDA, S.; IIDA, T.; SHIMADA, A.; SASAKI, K. Effects of cognitive behavioral therapy on orofacial pain conditions. Journal of Oral Science, v. 63, n. 1, p. 4-7, 2020. DOI: https://doi.org/10.2334/josnusd.20-0437.

OKESON, J. P. Tratamento das Desordens Temporomandibulares e Oclusão. Rio de Janeiro: GEN Guanabara Koogan, 2013.

PORTERO, P. P.; KERN, R.; KUSMA, S. Z.; GRAU-GRULLÓN, P. Placas oclusais no tratamento da disfunção temporomandibular (DTM). Revista Gestão \& Saúde, Curitiba, v. 1, n. 1, p. 36-40, 2009. Disponível em: http://www.herrero.com.br/files/revista/fileb5e3973b7f4f21920c93addd6d1853fb.pdf. Acesso em: 5 nov. 2021.

ROSAL, T. D. P. Disfunção temporomandibular em crianças: como diagnosticar? 2019. 6 f. Trabalho de Conclusão de Curso (Bacharelado em Odontologia) - Centro Universitário do Planalto Central Apparecido dos Santos, Brasília, 2019. Disponível em: https://dspace.uniceplac.edu.br/handle/123456789/254. Acesso em: 5 nov. 2021.

ROTA, A. C. Avaliação e implementação de nova forma de tratamento para a Disfunção

Temporomandibular. 2019. 94 f. Dissertação (Mestrado em Odontologia) - Faculdade de Ciências da Saúde Universidade de Brasília, Brasília, 2019. Disponível em: https://repositorio.unb.br/handle/10482/36731. Acesso em: 5 nov. 2021

STÜERMER, V. M. Avaliação da telessaúde no gerenciamento da disfunção temporomandibular na atenção primária à saúde. 2020. 35 f. Dissertação (Mestrado em Epidemiologia) - Faculdade de Medicina da UFRGSul, Rio Grande do Sul, 2020. Disponível em: http://hdl.handle.net/10183/217543. Acesso em: 5 nov. 2021.

TAKEUCHI-SATO, T.; ONO, Y.; FUNATO, M.; SATO, H.; SUGANUMA, T.; BABA, K. Efficacy of an email-based recording and reminding system for limiting daytime non-functional tooth contact in patients with temporomandibular disorders: A randomized controlled trial. Journal of oral rehabilitation, v. 47, n. 2, p. 158163, ago. 2020. Disponível em: https://onlinelibrary.wiley.com/doi/abs/10.1111/joor.12875. Acesso em: 5 nov. 2021.

TOSATO, J. D. P. Relação entre estresse, atividade muscular e disfunção temporomandibular. 2011.85 f. Tese (Doutorado em Odontologia) - Faculdade de Odontologia de Piracicaba da UNICAMP, Piracicaba, 2011.

\section{POLÊM!CA | LABORE}

Polêmica - Revista Eletrônica da Uerj - Rua São Francisco Xavier, 524, $1^{\circ}$ andar bloco D, sl.1001 • Tels.: +55 21 2334-4088 / 4087 • http://www.e-publicacoes.uerj.br/index.php/polemica/index http://www.labore.uerj.br • laboreuerj@yahoo.com.br 
Disponível em: http://repositorio.unicamp.br/handle/REPOSIP/288442. Acesso em: 5 nov. 2021.

URBANI, G.; JESUS, L. F. D.; COZENDEY-SILVA, E. N. Síndrome da disfunção da articulação temporomandibular e o estresse presente no trabalho policial: revisão integrativa. Ciência \& Saúde Coletiva, v. 24, n. 5, p. 1753-1765, maio 2019. DOI: https://doi.org/10.1590/1413-81232018245.16162017.

ZHANG, Q.; ZHANG, J.; RAN, W.; YU, S.; JIN, Y. Effectiveness of cognitive behavioral therapy on kinesiophobia and oral health-related quality of life in patients with temporomandibular disorders, study protocol for a randomized controlled trial. Medicine, v. 99, n. 47, p. 1-9, nov. 2020. Disponível em: https://www.ncbi.nlm.nih.gov/pmc/articles/PMC7676583/. Acesso em: 5 nov. 2021

ZAVANELLI, A. C.; REZENDE, M. C. R. A.; SANTOS-NETO, O. M.; FAJARDO, R. S. Integração da Psicologia e Odontologia na DTM: revisão sistematizada. Archives of Health Investigation, v. 6, n. 11, p. 530534, dez. 2017. DOI: https://doi.org/10.21270/archi.v6i11.2266.

Recebido em: 05/11/2021.

Aceito em: 30/11/2021.

\section{POLÊM!CA $\mid$ LABORE}

